\title{
Bounds for frequencies of class groups of real quadratic genus 1 function fields
}

\author{
by \\ Christian Friesen (Marion, OH)
}

1. Introduction. A long-standing conjecture of Gauss [11] is that there are infinitely many real quadratic number fields with ideal class number 1 and empirical evidence suggests the much stronger statement that about $3 / 4$ of all primes $p$ give rise to a field $\mathbb{Q}(\sqrt{p})$ with a class number of 1 . This observation, and many others, have been given a wonderful heuristic explanation by Cohen and Lenstra [4]. Although computational data is abundant $[2,3,14,17,20]$, proofs are conspicuously absent.

In the function field case, where similar conjectures have been introduced by Friedman and Washington [6] and refined by $\mathrm{Yu}$ [21], more is known. Let $\mathbb{F}_{q}$ be the finite field of $q$ elements, let $T$ be an indeterminate and let $M \in \mathbb{F}_{q}[T]$ be a squarefree monic polynomial of even degree $d$. Write the ideal class number of $\mathbb{F}_{q}(T, \sqrt{M(T)})$ as $h_{M}$. Although the expectations in this setting are frequently stated in terms requiring the degree to increase as $q$ stays fixed we will be fixing the degree and letting $q$ increase. Madan [15] showed that there are infinitely many $q$ such that there is an $M \in \mathbb{F}_{q}[T]$ with $h_{M}=1$ and $d=4$ and Schmidt [19] proved that for sufficiently large primes $q$ there exists an $M \in \mathbb{F}_{q}[T]$ with $d=6$ and $h_{M}=1$.

Suppose we are interested in arbitrary odd class numbers $h$. Ichimura [13] proved that there exist infinitely many primes $q$ with quartics $M \in \mathbb{F}_{q}[T]$ such that $h_{M}=h$. In a joint paper with van Wamelen [10] it was shown that for all odd $h$ and for all $q$ (a power of a prime $p \geq 5$ ) that are sufficiently large there exist quartics $M \in \mathbb{F}_{q}[T]$ such that $h_{M}=h$. In the same article it was shown that there are at least $q^{7 / 2} /(10 \log \log q)$ monic irreducible quartics $M \in \mathbb{F}_{q}[T]$ that satisfy $h_{M}=1$. This last result gives a lower bound of about $0.4 q^{-1 / 2}(\log \log q)^{-1}$ for the probability that a randomly chosen irreducible monic quartic $M \in \mathbb{F}_{q}[T]$ has ideal class number 1 .

2000 Mathematics Subject Classification: Primary 11R29; Secondary 11G20, 11R58.

Key words and phrases: class groups, Cohen-Lenstra conjecture, function fields. 
This is somewhat unsatisfying as this lower bound vanishes when $q \rightarrow \infty$ whereas the empirical data [7] quite clearly indicates that we should expect proportions close to $3 / 4$. As one of our main results we prove here that, when constrained to those monic quartics that have an irreducible cubic factor, the proportion of $M \in \mathbb{F}_{q}[T]$ with $h_{M}=1$ is between $70 \%$ and $84 \%$.

Further motivation for looking at ideal class groups in this context comes from the field of cryptography. Scheidler, Stein and Williams [18] have proposed a key-exchange cryptosystem based on the continued fraction expansion of an irrational quadratic in a real quadratic function field $\mathbb{F}_{q}(T, \sqrt{M(T)})$. The case where $M$ has degree $d=4$ was chosen as the one that performed best in terms of empirically-measured speed vs. hypothesized security. The security of this cryptosystem hinges on two main conjectures: that the discrete logarithm problem is usually difficult and that the continued fraction expansion of $\mathbb{F}_{q}(T, \sqrt{M(T)})$ is usually large (or, equivalently, that the ideal class number is small). Showing, as we will, that the class number is 1 more than $2 / 3$ of the time means that the second assumption above will not be of concern as one could repeatedly iterate the coding process with enough different values of $M$ to obtain arbitrarily small probability that none of them have class number of 1 . This leaves us with a probability arbitrarily close to 1 that the code will not be broken due to a small continued fraction expansion.

The question as to how often we should expect the ideal class number to be divisible by a prime $p$ is also of some interest. Restricting ourselves once more to the function field case we have a result of $\mathrm{Yu}$ [21] where for a fixed degree $d$ the fraction of ideal class groups with a given $p$-Sylow group tends towards some limit as $q \rightarrow \infty$, subject to the condition that $p \nmid q-1$. $\mathrm{Yu}$ further shows that the limit (as $d \rightarrow \infty$ with $d$ even) of these limits exists and is equal to that predicted by the Cohen-Lenstra heuristics! The values of the individual limits, however, (say for $d=4$, which is the case of interest for this paper) remain unspecified. We shall shed light on some of the behavior in the $d=4$ case by determining upper and lower bounds for $P\left(h_{M} \equiv 0(\bmod p)\right)$ and $P\left(h_{M}=p\right)$ for odd primes $p$. We shall also investigate how often the $p$-rank of the ideal class group is equal to 2 .

2. Preliminaries. Let $q$ be a prime, $\mathbb{F}_{q}$ be the finite field of $q$ elements and $T$ be an indeterminate. For any squarefree monic quartic $M \in \mathbb{F}_{q}[T]$ we write the ideal class group of $\mathbb{F}_{q}(T, \sqrt{M(T)})$ as $\mathcal{C} l_{M}$ and the cardinality of $\mathcal{C} l_{M}$, the ideal class number, as $h_{M}$. Readers interested in an introduction to quadratic function fields are directed to Emil Artin's thesis [1] or to more recent work of Hayes [12].

Define $S_{q}$ to be the set of all monic quartics $M \in \mathbb{F}_{q}[T]$ that are divisible by an irreducible cubic in $\mathbb{F}_{q}[T]$. For the remainder of the paper we will 
restrict our attention solely to polynomials belonging in this set - a restriction that is forced on us by the nature of some results that we pilfer from an earlier paper. At times it will be useful to consider the subset $S_{q}^{\prime}$, defined as those elements of $S_{q}$ with a zero coefficient for the cubic term (i.e. those of the form $M(T)=T^{4}+a T^{2}+b T+c$ for some $\left.a, b, c \in \mathbb{F}_{q}\right)$. For any $N(T) \in S_{q}^{\prime}$ consider the translates of $N$, those $M \in S_{q}$ of the form $M(T)=N(T+d)$ where $d \in \mathbb{F}_{q}$. Since $\mathbb{F}_{q}(T, \sqrt{N(T)})$ and $\mathbb{F}_{q}(T, \sqrt{M(T)})$ are isomorphic via $T \mapsto T+d$ and $\sqrt{N(T)} \mapsto \sqrt{M(T)}$ it follows that $\mathcal{C} l_{M} \cong \mathcal{C} l_{N}$ for any translate $M$ of $N$. Therefore any result concerning the proportions of ideal class groups in $S_{q}^{\prime}$ will automatically be true also of the ideal class groups of $S_{q}$.

Suppose that $M(x)$ is a monic squarefree quartic for which the Jacobian of $y^{2}=M(x)$ has odd cardinality. All points on this curve not of the form $(x, 0)$ can be paired up via $(x, y) \leftrightarrow(x,-y)$. This implies that there are an odd number of points on the curve precisely when there are an odd number of solutions to $M(x)=0$. For a squarefree quartic this is equivalent to saying that $M$ is the product of a linear polynomial and a cubic irreducible polynomial; in other words the Jacobian of $y^{2}=M(x)$ has odd cardinality if and only if $M \in S_{q}$. This will, as a consequence later on, restrict our attention to those elliptic curves whose elliptic groups are also of odd cardinality.

We shall be somewhat relaxed with our terminology and write, for instance,

$$
P(h \equiv 0(\bmod p)) \quad \text { to mean } \quad \frac{\#\left\{M \in S_{q}: h_{M} \equiv 0(\bmod p)\right\}}{\# S_{q}} .
$$

The number of monic irreducible cubics in $\mathbb{F}_{q}[T]$ is given by $\left(q^{3}-q\right) / 3$ and there are $q$ linear polynomials so $\# S_{q}=\left(q^{4}-q^{2}\right) / 3$. Define $N_{q}(A, B)$ as the number of isomorphism classes of elliptic curves over $\mathbb{F}_{q}$ with $E\left(\mathbb{F}_{q}\right) \cong$ $C_{A} \times C_{B}$.

Before submerging ourselves in the proof details it would, perhaps, be appropriate to outline our approach. Suppose, for instance, that we wish to bound $P(h \equiv 0(\bmod 3))$. We would begin with the bounds for the $N_{q}(A, B)$ that were obtained in a prior paper [8] and sum over all $A \equiv 0(\bmod 3)$ to obtain the number of desired isomorphism classes. Next we would determine the number of elliptic curves of the form $E: y^{2}=x^{3}+R x+S$ in each isomorphism class. Then, for each such curve $E$, we would count the number of $\mathbb{F}_{q}$-rational points $\mathcal{P}$ such that the subgroup $E\left(\mathbb{F}_{q}\right) /\langle\mathcal{P}\rangle$ satisfies the criteria for our ideal class groups (in this particular example we would require that the subgroup has cardinality divisible by 3 ). We would then see a 1-1 correspondence between such $E, \mathcal{P}$ pairs and $M \in S_{q}^{\prime}$ under which $\mathcal{C} l_{M} \cong E\left(\mathbb{F}_{q}\right) /\langle\mathcal{P}\rangle$. It is precisely at this step that our decision to 
examine the set $S_{q}^{\prime}$ forces us to consider only those elliptic groups where $\# E\left(\mathbb{F}_{q}\right)=A B$ is odd. We then multiply our count by $q$, accounting for translations, to obtain the number of desired $M \in S_{q}$ and finally divide by the total number of $M \in S_{q}$ to arrive at the proportion of $M$ with the desired class group structure.

Without further ado we introduce some necessary notation. We write $m \mid n$ to mean that $m$ divides $n$ and $m \| n$ to mean that $m \mid n$ and $(m, n / m)=$ 1. Also useful to us will be $\lfloor x\rfloor$ which denotes the greatest integer less than or equal to $x . C_{n}$ shall be the cyclic group of order $n$ and, for any set $S$, we use $\# S$ for the cardinality of $S$. Let $\operatorname{ord}_{p}(v)$ be the highest power of $p$ dividing $v$. For $q \geq 3$ a prime and $v$ and $w$ odd positive integers with $w \mid q-1$ we define

$$
\sigma(v)=\prod_{\substack{p^{2}|v \\ p|(q-1) / w}} p^{\left\lfloor\operatorname{ord}_{p}(v) / 2\right\rfloor}
$$

From a previous paper [8] we have the following:

THEOREM. Let $q>e^{100}$ be an odd prime. Let $v$ and $w$ be positive odd integers such that $w \mid q-1$. Define $\tau(x)$ as the number of positive squarefree divisors of $x$ and let $\phi()$ denote Euler's totient function. Define $\sigma(v)$ as above. For any odd positive integers $A$ and $B$ with $B \mid A$ define $N_{q}(A, B)$ as the number of isomorphism classes of elliptic curves, $E$, over $\mathbb{F}_{q}$ with $E\left(\mathbb{F}_{q}\right) \cong C_{A} \times C_{B}$ where $C_{n}$ is the cyclic group of order $n$. Then

$$
\begin{aligned}
& \sum_{\substack{B \equiv 0(\bmod w) \\
A B \equiv 0\left(\bmod v w^{2}\right) \\
A B \text { odd }}} N_{q}(A, B) \\
& \quad<\frac{0.74 q}{\phi(v) w^{3}}\left(\prod_{\substack{p \| v \\
p \mid(q-1) / w}} \frac{p}{p+1}\right)\left(1+\frac{132 w^{1.25} \tau(v) v \sigma(v)}{q^{0.125}}\right) .
\end{aligned}
$$

When, in addition, $v w<q^{0.07}$ we also have

$$
\begin{aligned}
& \sum_{\substack{B \equiv 0(\bmod w) \\
A B \equiv 0\left(\bmod v w^{2}\right) \\
A B \text { odd }}} N_{q}(A, B) \\
& \quad>\frac{0.58 q}{\phi(v) w^{3}}\left(\prod_{\substack{p|v \\
p|(q-1) / w}} \frac{p}{p+1}\right)\left(1-\frac{132 w^{1.25} \tau(v) v}{q^{0.125}}\right) .
\end{aligned}
$$


REMARK. If $w \nmid q-1$ then

$$
\sum_{\substack{B \equiv 0(\bmod w) \\ A \equiv 0\left(\bmod v w^{2}\right) \\ A B \text { odd }}} N_{q}(A, B)=0 .
$$

This is equivalent to the statement that if we have an elliptic curve with $E\left(\mathbb{F}_{q}\right) \cong C_{A} \times C_{B}$ and $B \mid A$ then we must also have $B \mid q-1$.

We now wish to determine the number of elliptic curves of the form $E: y^{2}=x^{3}+R x+S$ in each of these isomorphism classes. From earlier comments we are only interested in non-singular elliptic curves with groups of odd cardinality. Since the only points that are not paired up via $(x, y) \leftrightarrow$ $(x,-y)$ are those with $y=0$ or the point at infinity it follows that we must have an even number of solutions to $0=x^{3}+R x+S$. This, in turn, means that $x^{3}+R x+S$ is an irreducible cubic and as a consequence we must have $S \neq 0$. If $q \not \equiv 1(\bmod 3)$ then $R$ must be non-zero if $x^{3}+R x+S$ is irreducible. Two elliptic curves, $E_{1}$ and $E_{2}$, of the form $E_{i}: y^{2}=x^{3}+R_{i} x+S_{i}$ are isomorphic if and only if $R_{2}=a^{4} R_{1}$ and $S_{2}=a^{6} S_{1}$ for some $a \in \mathbb{F}_{q}^{*}$. With the exception of at most 4 isomorphism classes (characterized by $R=0$ when $q \equiv 1(\bmod 3))$ we have exactly $(q-1) / 2$ elliptic curves of the desired form in each isomorphism class. The exceptional classes, when they occur, have exactly $(q-1) / 6$ elliptic curves of the desired form.

It follows that, for any odd $A$ and $B$, the number of elliptic curves of the form $E: y^{2}=x^{3}+R x+S$ with $E\left(\mathbb{F}_{q}\right) \cong C_{A} \times C_{B}$ is at least

and at most

$$
\left(N_{q}(A, B)-4\right) \frac{q-1}{2}+4 \frac{q-1}{6}>\left(N_{q}(A, B)-3\right) \frac{q-1}{2}
$$

$$
N_{q}(A, B) \frac{q-1}{2} \text {. }
$$

We paraphrase without proof an earlier result [9], Theorem 2.5, to obtain the following theorem.

THEOREM 2.3. Let $\mathbb{F}_{q}$ be the finite field with $q$ elements and characteristic $\neq 2,3$. There is a 1-1 correspondence between $M \in S_{q}^{\prime}$ and pairs $E, \mathcal{P}$ of non-singular elliptic curves $E: w^{2}=v^{3}+A v+B$ with $\# E\left(\mathbb{F}_{q}\right)$ odd and with $\mathcal{P}$ a finite $\mathbb{F}_{q}$-rational point on $E$. Under this correspondence the ideal class group of $\mathbb{F}_{q}(T, \sqrt{M(T)})$ is isomorphic to the coset $E\left(\mathbb{F}_{q}\right) /\langle\mathcal{P}\rangle$ where $\langle\mathcal{P}\rangle$ denotes the subgroup generated by $\mathcal{P}$.

The above theorem is a consequence of a birational equivalence between an elliptic curve, $E$, and a plane quartic model, $y=M(x)$, for it. Under such a correspondence the Jacobian of the quartic may be canonically identified with the group $E\left(\mathbb{F}_{q}\right)$ and it is this correspondence that leads to the stated result. 
Remark. Suppose that $E\left(\mathbb{F}_{q}\right) \cong C_{A} \times C_{B}$ with an odd prime $p \mid A$ and $p \nmid B$. Then there exist $A B / p$ elements $g$ of $C_{A} \times C_{B}$ with $p \mid(A B / \#\langle g\rangle)$. Since one of these elements $g$ is the identity, corresponding to the point at infinity of $E\left(\mathbb{F}_{q}\right)$, there are exactly $A B / p-1$ finite $\mathbb{F}_{q}$-rational points $\mathcal{P}$ on $E$ that will have associated quartics in $S_{q}^{\prime}$ with ideal class group divisible by $p$.

The Weil bound gives $\# E\left(\mathbb{F}_{q}\right) \in\left[(\sqrt{q}-1)^{2},(\sqrt{q}+1)^{2}\right]$ (this standard result can be found, for instance, in Eichler [5]) so the number of finite $\mathbb{F}_{q}$-rational points $\mathcal{P}$ for each curve $E$ is in $[q-2 \sqrt{q}, q+2 \sqrt{q}]$.

3. Bounds on ideal class group frequencies. The author has some conjectures [7] for the degree 4 case which are in close, but not perfect, agreement with those obtained by averaging the Cohen-Lenstra heuristics. As a consequence, it is conjectured that for $q \not \equiv 1(\bmod p)$ we should see that

$$
\lim _{q \rightarrow \infty} P\left(h_{M} \equiv 0(\bmod p)\right)=\frac{1}{p(p-1)}
$$

with slightly different limits if we restrict our $q$ to those satisfying $q \equiv 1$ $(\bmod p)$. In the following theorem we prove upper and lower bounds that differ from the above prediction by less than $20 \%$ (in relative terms).

Theorem 3.1. Let $q>e^{100}$ be prime. Let $p$ be an odd prime. Define $S_{q}$ to be the set of all monic quartics $M \in \mathbb{F}_{q}[T]$ that are divisible by an irreducible cubic in $\mathbb{F}_{q}[T]$. Let $h_{M}$ be the ideal class number of $\mathbb{F}_{q}(T, \sqrt{M(T)})$ and write $P(p \mid h)$ as shorthand for $\#\left\{M \in S_{q}: h_{M} \equiv 0(\bmod p)\right\} / \# S_{q}$. Then

$$
P(p \mid h)<\frac{1.12}{p(p-1)}+\frac{295}{q^{0.125} p}+\frac{232}{q^{0.125} p^{1.75}} .
$$

If, in addition to the above hypotheses, we require that $p<q^{0.03}$, then we obtain the lower bounds

$$
\begin{array}{ll}
P(p \mid h)>\frac{0.867}{p(p-1)}-\frac{234}{q^{0.125} p}>\frac{0.84}{p(p-1)} & \text { if } p \nmid q-1, \\
P(p \mid h)>\frac{0.867(p+1)}{p^{3}}-\frac{234}{q^{0.125} p}-\frac{150}{q^{0.125} p^{1.75}}>\frac{0.84(p+1)}{p^{3}} & \text { if } p \mid q-1 .
\end{array}
$$

Proof. If we wish the ideal class group of the quartic to be divisible by $p$ then $p$ must divide the order of group of the associated elliptic curve. This is only possible if $p \mid A$ where $E\left(\mathbb{F}_{q}\right) \cong C_{A} \times C_{B}$. We begin by considering the case where $p \nmid q-1$. Recalling formula (2.1) and setting $v=p$ and $w=1$ we obtain

$$
\sum_{\substack{A \equiv 0(\bmod p) \\ A \text { odd }}} N_{q}(A, B)=\sum_{\substack{A B \equiv 0(\bmod p) \\ A B \text { odd }}} N_{q}(A, B)<q\left(\frac{0.74}{p-1}+\frac{196 p}{q^{0.125}(p-1)}\right) .
$$


Now, each isomorphism class contains at most $(q-1) / 2$ elliptic curves of the desired form and each such curve has a group of order less than $q+2 \sqrt{q}+1$. Since $p \nmid q-1$ it follows from the remark immediately after (2.2) that $p \nmid B$. The remark following Theorem 2.3 implies that, for each such elliptic curve, just less than 1 in $p$ of its $\mathbb{F}_{q}$-rational points $\mathcal{P}$ will result in a quartic with an ideal class number divisible by $p$. Therefore the number of monic quartics $M \in S_{q}^{\prime}$ that give rise to ideal class numbers of $\mathbb{F}_{q}(T, \sqrt{M(T)})$ that are divisible by $p$ is bounded from above by

$$
q\left(\frac{0.74}{p-1}+\frac{196 p}{q^{0.125}(p-1)}\right) \frac{q-1}{2} \cdot \frac{q+2 \sqrt{q}+1}{p}<q^{3}\left(\frac{0.37}{p^{2}-p}+\frac{98}{q^{0.125} p}\right) .
$$

Let us now consider the other case, where $p \mid q-1$. Again referring to (2.1) with $v=p$ and $w=1$ we obtain the following bound for the number of desired isomorphism classes:

$$
\sum_{\substack{A \equiv 0(\bmod p) \\ A \text { odd }}} N_{q}(A, B)<q\left(\frac{0.74 p}{p^{2}-1}+\frac{196 p^{2}}{q^{0.125}\left(p^{2}-1\right)}\right) .
$$

Some of isomorphism classes above will have a $p$-rank of 2 . We determine an upper bound for these via (2.1) once again, this time with $v=1$ and $w=p$. The number of isomorphism classes with a $p$-rank of 2 is then bounded from above by

$$
\sum_{\substack{A \equiv 0(\bmod p) \\ B \equiv 0(\bmod p) \\ A B \operatorname{odd}}} N_{q}(A, B)=\sum_{\substack{B \equiv 0(\bmod p) \\ A B \equiv 0\left(\bmod p^{2}\right) \\ A B \text { odd }}} N_{q}(A, B)<q\left(\frac{0.74}{p^{3}}+\frac{98}{q^{0.125} p^{1.75}}\right) .
$$

If an elliptic curve $E$ has a $p$-rank of 2 then $p \mid \#\left(E\left(\mathbb{F}_{q}\right) /\langle\mathcal{P}\rangle\right)$ for all $\mathcal{P}$. It follows that every $\mathbb{F}_{q}$-rational point $\mathcal{P}$ of $E$ gives rise to a quartic $M$ with $p \mid h_{M}$. So, we shall count, with weight $1 / p$, all the elliptic curves with $p$-rank at least 1 and then count, with weight $1-1 / p$, those with $p$-rank of 2 and thus avoid doubly counting the same contribution. We obtain the following upper bound for the number of monic quartics $M \in S_{q}^{\prime}$ that give rise to an ideal class group divisible by $p$ :

$$
\begin{gathered}
q^{3}\left(\frac{0.37}{p^{2}-1}+\frac{98 p}{q^{0.125}\left(p^{2}-1\right)}+\left(1-\frac{1}{p}\right)\left(\frac{0.37}{p^{3}}+\frac{49}{q^{0.125} p^{1.75}}\right)\right) \\
<q^{3}\left(0.37\left(\frac{1}{p^{2}-1}+\frac{p-1}{p^{4}}\right)+\frac{98 p}{q^{0.125}\left(p^{2}-1\right)}+\frac{49(p-1)}{q^{0.125} p^{2.75}}\right) \\
<q^{3}\left(\frac{0.37}{p(p-1)}+\frac{98}{q^{0.125} p}+\frac{77}{q^{0.125} p^{1.75}}\right) .
\end{gathered}
$$


As this bound is larger (with the presence of the third term) than the bound in the case where $p \nmid q-1$ we conclude that, no matter what the congruence of $q$ is modulo $p$, we have an upper bound for the number of monic quartics $M \in S_{q}^{\prime}$ having an ideal class number of $\mathbb{F}_{q}(T, \sqrt{M(T)})$ divisible by $p$ of

$$
q^{3}\left(\frac{1.12}{p(p-1)}+\frac{295}{q^{0.125} p}+\frac{232}{q^{0.125} p^{1.75}}\right) .
$$

From our previous remark concerning $S_{q}$ and $S_{q}^{\prime}$ we may multiply these results for $S_{q}^{\prime}$ by $q$ to obtain the desired upper bounds for the set $S_{q}$.

We begin our proof of the lower bounds by first treating the case $p \nmid q-1$. From (2.2), with $v=p$ and $w=1$ we have

$$
\sum_{\substack{A \equiv 0(\bmod p) \\ A \text { odd }}} N_{q}(A, B)=\sum_{\substack{A B \equiv 0(\bmod p) \\ A B \text { odd }}} N_{q}(A, B)>q\left(\frac{0.58}{p-1}-\frac{154 w^{1.25} p}{q^{0.125}(p-1)}\right) .
$$

We see that there are at least

$$
q\left(\frac{0.58}{p-1}-\frac{154 w^{1.25} p}{q^{0.125}(p-1)}-\frac{3}{q}\right) \frac{q-1}{2}
$$

elliptic curves of the desired form with $p \mid A$ each of which has at least $q-2 \sqrt{q}+1$ points $\mathcal{P}$ on it. Since $p \nmid q-1$ the $p$-Sylow subgroup is cyclic and therefore there are $\# E / p-1$ points $\mathcal{P}$ on the curve that are associated with a quartic $M$ such that $h_{M} \equiv 0(\bmod p)$. It follows that the number of $M \in S_{q}^{\prime}$ with $p \mid h_{M}$ must be at least

$$
q^{3}\left(\frac{0.289}{p(p-1)}-\frac{78}{q^{0.125} p}\right)
$$

from which we can easily obtain the stated lower bounds for the case where $p \nmid q-1$.

The case $p \mid q-1$ is more complicated. If the $p$-rank of the elliptic group is 1 then we proceed as before. If $E\left(\mathbb{F}_{q}\right) \cong C_{A} \times C_{B}$ with $p \mid B$ then every associated monic will have an ideal class group divisible by $p$.

From (2.2), with $v=p$ and $w=1$ we have

$$
\sum_{\substack{A \equiv 0(\bmod p) \\ A \text { odd }}} N_{q}(A, B)>q\left(\frac{0.58 p}{p^{2}-1}-\frac{154 p^{2}}{q^{0.125}\left(p^{2}-1\right)}\right) .
$$

If all of the elliptic curves in the isomorphism classes counted above had a $p$-rank of 1 then we would proceed as before, with the comment that the number of $M$ in $S_{q}^{\prime}$ related to each such curve must be $\# E\left(\mathbb{F}_{q}\right) / p-1$. However, since we are in the case where $p \mid q-1$, some of the isomorphism classes will have elliptic curves with a $p$-rank of 2 and for these curves every one of the associated $M \in S_{q}^{\prime}$ will satisfy $h_{M} \equiv 0(\bmod p)$. To determine the 
additional contribution from these we find a lower bound for the number of isomorphism classes with a $p$-rank of 2 by setting $v=1$ and $w=p$ in (2.2) to obtain

$$
\sum_{\substack{B \equiv 0(\bmod p) \\ A B \equiv 0\left(\bmod p^{2}\right) \\ A B \text { odd }}} N_{q}(A, B)>q\left(\frac{0.58}{p^{3}}-\frac{77 p^{1.25}}{q^{0.125} p^{3}}\right) .
$$

Combining the contributions from the elliptic curves of $p$-rank 1 with those of $p$-rank 2 , being careful not to count the same curves twice, gives us the following lower bound for the number of $M \in S_{q}^{\prime}$ with $p \mid h_{M}$ :

$$
\begin{aligned}
\#\left\{M \in S_{q}^{\prime}:\right. & \left.h_{M} \equiv 0(\bmod p)\right\} \\
> & \frac{q(q-1)}{2}\left(\frac{0.58 p}{p^{2}-1}-\frac{154 p^{2}}{q^{0.125}\left(p^{2}-1\right)}\right) \frac{q-2 \sqrt{q}+1-p}{p} \\
& +\frac{q(q-1)}{2}\left(\frac{0.58(p-1)}{p^{4}}-\frac{77 p^{1.25}(p-1)}{q^{0.125} p^{4}}\right)(q-2 \sqrt{q}) \\
> & q^{3}\left(\frac{0.289(p+1)}{p^{3}}-\frac{78}{q^{0.125} p}-\frac{50}{q^{0.125} p^{1.75}}\right) .
\end{aligned}
$$

Multiplying the above result by $q$ converts it to the number of $M \in S_{q}$ with $p \mid h_{M}$. Finally, since $\# S_{q}<q^{4} / 3$, if we further divide by $q^{4} / 3$ we will immediately obtain the lower bound for $P(h \equiv 0(\bmod p))$ in the case where $p \mid q-1$, concluding our proof.

Lemma 3.2. Let $q>e^{100}$ be prime. Let $p$ and $r$ be distinct odd primes. Define $S_{q}$ to be the set of all monic quartics $M \in \mathbb{F}_{q}[T]$ that are divisible by an irreducible cubic in $\mathbb{F}_{q}[T]$. Let $h_{M}$ be the ideal class number of $\mathbb{F}_{q}(T, \sqrt{M(T)})$ and write $P(p r \mid h)$ as shorthand for $\#\left\{M \in S_{q}: h_{M} \equiv 0\right.$ $(\bmod p r)\} / \# S_{q}$. Then, for $M \in S_{q}$,

$$
P(p r \mid h)<\frac{1.12}{\left(p^{2}-p\right)\left(r^{2}-r\right)}+\frac{597}{q^{0.125}(p-1)(r-1)} .
$$

Proof. If we wish the ideal class group of the quartic to be divisible by $p r$ then $p r$ must divide the order of the group of the associated elliptic curve. We treat separately the 4 cases depending on the value of $\operatorname{gcd}(p r, q-1)$ and begin by considering the case where $\operatorname{gcd}(p r, q-1)=1$. Recalling the result of (2.1) and setting $v=p r$ and $w=1$ we obtain

$$
\sum_{\substack{A B \equiv 0(\bmod p r) \\ A B \text { odd }}} N_{q}(A, B)<\frac{0.74 q}{(p-1)(r-1)}\left(1+\frac{528 p r}{q^{0.125}}\right) .
$$

Now, each isomorphism class contains at most $(q-1) / 2$ elliptic curves of the desired form and each such curve has a group of order less than 
$q+2 \sqrt{q}+1$. Since $p \nmid q-1$ and $r \nmid q-1$ it follows that $p \nmid B$ and $r \nmid B$ and we have $\# E\left(\mathbb{F}_{q}\right) /(p r)-1$ points $\mathcal{P}$ such that the quartic $M$ associated with $(E, \mathcal{P})$ satisfies $h_{M} \equiv 0(\bmod p r)$. Therefore the number of monic quartics $M \in S_{q}^{\prime}$ with $p r \mid h_{M}$ is bounded from above by

$$
\begin{aligned}
q \frac{0.74}{(p-1)(r-1)}\left(1+\frac{528 p r}{q^{0.125}}\right) \frac{q-1}{2} & \cdot \frac{q+2 \sqrt{q}+1}{p r} \\
<q^{3} \frac{0.37}{p r(p-1)(r-1)} & \left(1+\frac{528 p r}{q^{0.125}}\right) .
\end{aligned}
$$

Dividing this by $\# S_{q}^{\prime}$ gives, for the case where $\operatorname{gcd}(p r, q-1)=1$,

$$
P(p r \mid h)<\frac{1.12}{\left(p^{2}-p\right)\left(r^{2}-r\right)}+\frac{587}{q^{0.125}(p-1)(r-1)} .
$$

The next cases to be considered are when exactly one of $p$ and $r$ divides $q-1$. We shall treat the case where $\operatorname{gcd}(p r, q-1)=p$ and use the symmetry of the situation to obtain the other result as well. We shall see that there are two main differences between this case and the situation where $\operatorname{gcd}(p r, q-1)$ $=1$. They are the presence of the factor $p /(p+1)$ that shows up in the determination of the number of isomorphism classes and the fact that some of the elliptic curves will now have a $p$-rank of 2 .

Referring to (2.1) once again we obtain the bound below for the number of isomorphism classes of desired form:

$$
\sum_{\substack{A B \equiv 0(\bmod p r) \\ A B \text { odd }}} N_{q}(A, B)<\frac{0.74 q p}{\left(p^{2}-1\right)(r-1)}\left(1+\frac{528 p r}{q^{0.125}}\right) .
$$

Some of the isomorphism classes above will have a $p$-rank of 2 . We determine an upper bound for these via (2.1) once again, this time with $v=r$ and $w=p$. The number of isomorphism classes with a $p$-rank of 2 and divisible by $r$ is bounded from above by

$$
\sum_{\substack{B \equiv 0(\bmod p) \\ A B \equiv 0\left(\bmod p^{2} r\right) \\ A B \text { odd }}} N_{q}(A, B)<\frac{0.74 q}{(r-1) p^{3}}\left(1+\frac{264 p^{1.25} r}{q^{0.125}}\right) .
$$

All of the $\mathbb{F}_{q}$-rational points $\mathcal{P}$ on the elliptic curves $E$ with $p$-rank of 2 give rise to quartics with associated ideal class number divisible by $p$ and fewer than $\# E\left(\mathbb{F}_{q}\right) / r$ of these give class numbers that are also divisible by $r$. To avoid counting the same curve twice we add to the amount from the curves with $p$-rank $\geq 1$ a correction equal to $(p-1) / p$ of the total we obtain from the $p$-rank 2 curves. We obtain the following upper bound, when $\operatorname{gcd}(p r, q-1)=p$, for the number of monic quartics $M \in S_{q}^{\prime}$ such that $h_{M}$ 
is divisible by $p r$ :

$$
\begin{aligned}
& q^{3}\left(\frac{0.37}{\left(p^{2}-1\right)\left(r^{2}-r\right)}\left(1+\frac{528 p r}{q^{0.125}}\right)+\frac{0.37(p-1)}{\left(r^{2}-r\right) p^{4}}\left(1+\frac{264 p^{1.25} r}{q^{0.125}}\right)\right) \\
&<q^{3}\left(0.37\left(\frac{p^{4}+p^{3}-p^{2}-p+1}{p^{4}\left(p^{2}-1\right)\left(r^{2}-r\right)}\right)\right. \\
&\left.+\frac{196 p r}{q^{0.125}\left(p^{2}-1\right)\left(r^{2}-r\right)}+\frac{98(p-1) p^{1.25} r}{q^{0.125}\left(r^{2}-r\right) p^{4}}\right) \\
&< q^{3}\left(\frac{0.37}{\left(p^{2}-p\right)\left(r^{2}-r\right)}+\frac{198}{q^{0.125}(p-1)(r-1)}\right)
\end{aligned}
$$

where we have used basic calculus to see that

$$
\frac{196 p}{p^{2}-1}+\frac{98(p-1)}{p^{2.75}}<\frac{198}{p-1}
$$

Since the bound we have obtained above is symmetric with respect to $p$ and $r$ it follows that the same upper bound (for the number of monic quartics $M \in S_{q}^{\prime}$ such that $h_{M}$ is divisible by $p r$ ) holds for the case where $p \nmid q-1$ and $r \mid q-1$.

The final case remaining is the case where $\operatorname{gcd}(p r, q-1)=p r$. We begin by determining an upper bound for the number of isomorphism classes whose groups are divisible by $p r$ and obtain

$$
\sum_{\substack{A B \equiv 0(\bmod p r) \\ A B \text { odd }}} N_{q}(A, B)<\frac{0.74 q p r}{\left(p^{2}-1\right)\left(r^{2}-1\right)}\left(1+\frac{528 p r}{q^{0.125}}\right) .
$$

We now need to determine the maximum number of isomorphism classes where the $p$-rank is 2 , where the $r$-rank is 2 and where they are both equal to 2 and sum their contributions making particular note of the fact that if both $p$ - and $r$-ranks of an elliptic curve are 2 then all associated values of $M \in S_{q}^{\prime}$ have $p r \mid h$. We obtain, for the number of monic quartics $M \in S_{q}^{\prime}$ such that $h_{M}$ is divisible by $p r$, the following upper bound:

$$
\begin{aligned}
& \frac{0.37 q^{3}}{\left(p^{2}-1\right)\left(r^{2}-1\right)}\left(1+\frac{528 p r}{q^{0.125}}\right)+\frac{p-1}{p r} \cdot \frac{0.37 q^{3}}{(r-1) p^{3}} \cdot \frac{r}{r+1}\left(1+\frac{264 p^{1.25} r}{q^{0.125}}\right) \\
& \quad+\frac{r-1}{p r} \cdot \frac{0.37 q^{3}}{(p-1) r^{3}} \cdot \frac{p}{p+1}\left(1+\frac{264 r^{1.25} p}{q^{0.125}}\right)+\frac{0.37 q^{3}}{p^{3} r^{3}}\left(1+\frac{132 p^{1.25} r^{1.25}}{q^{0.125}}\right) \\
& <0.37 q^{3}\left(\frac{1}{\left(p^{2}-1\right)\left(r^{2}-1\right)}+\frac{p-1}{\left(r^{2}-1\right) p^{4}}+\frac{r-1}{\left(p^{2}-1\right) r^{4}}+\frac{1}{p^{3} r^{3}}\right) \\
& +\frac{49 q^{3}}{q^{0.125}}\left(\frac{4 p r}{\left(p^{2}-1\right)\left(r^{2}-1\right)}+\frac{2 p^{1.25} r(p-1)}{\left(r^{2}-1\right) p^{4}}\right.
\end{aligned}
$$




$$
\begin{aligned}
& \left.+\frac{2 r^{1.25} p(r-1)}{\left(p^{2}-1\right) r^{4}}+\frac{p^{1.25} r^{1.25}}{p^{3} r^{3}}\right) \\
< & q^{3}\left(\frac{0.37}{\left(p^{2}-p\right)\left(r^{2}-r\right)}+\frac{199}{q^{0.125}(p-1)(r-1)}\right) .
\end{aligned}
$$

Note that, in all 4 cases, the number of desired monics $M \in S_{q}^{\prime}$ is less than

$$
q^{3}\left(\frac{0.37}{\left(p^{2}-p\right)\left(r^{2}-r\right)}+\frac{199}{q^{0.125}(p-1)(r-1)}\right)
$$

and that, once we divide this by $\left(q^{3}-q\right) / 3$ we can obtain the desired upper bound for the proportion of $M \in S_{q}^{\prime}$ such that $p r \mid h$. This proportion is necessarily the same when we consider $M \in S_{q}$ as well.

TheOREM 3.3. Let $q>e^{100}$ be prime. Let $p<q^{0.03}$ be an odd prime. Define $S_{q}$ to be the set of all monic quartics $M \in \mathbb{F}_{q}[T]$ that are divisible by an irreducible cubic in $\mathbb{F}_{q}[T]$. Let $h_{M}$ be the ideal class number of $\mathbb{F}_{q}(T, \sqrt{M(T)})$ and write $P(h=p)$ as shorthand for $\#\left\{M \in S_{q}: h_{M}=\right.$ $p\} / \# S_{q}$. Then, if $p \nmid q-1$,

$$
\frac{1}{p^{2}-p}\left(0.56-\frac{0.236 p}{q^{0.03}}+\frac{1.12}{p^{2}(p-1)}\right)<P(h=p)<\frac{1.121}{p(p-1)}
$$

and, if $p \mid q-1$,

$$
\frac{1}{p^{2}-p}\left(0.559-\frac{0.263 p}{q^{0.03}}-\frac{0.867}{p^{2}}+\frac{1.12}{p^{3}(p-1)}\right)<P(h=p)<\frac{1.121}{p(p-1)} .
$$

Pr o of. The trivial inequality $P(h=p) \leq P(h \equiv 0(\bmod p))$ together with Theorem 3.1 and the remark that

$$
\frac{295}{q^{0.125} p}+\frac{232}{q^{0.125} p^{1.75}}<0.0005
$$

serves to prove the upper bounds. The lower bounds, however, will require some effort. Let us begin with the case $p \nmid q-1$. It is clear that

$$
P(h=p) \geq P(h \equiv 0(\bmod p))-P\left(h \equiv 0\left(\bmod p^{2}\right)\right)-\sum_{\text {odd prime } r \neq p} P(p r \mid h) .
$$

The first term on the right has a lower bound of $\frac{0.867}{p(p-1)}-\frac{234}{q^{0.125} p}$ given by Theorem 3.1. The final summation can be bounded using Lemma 3.2 as follows:

$$
\sum_{\text {odd prime } r \neq p} P(p r \mid h)<\frac{1.12}{p^{2}-p} \sum_{r<q} \frac{1}{r^{2}-r}+\frac{597}{q^{0.125}(p-1)} \sum_{r<q}\left(\frac{1}{r}+\frac{1}{r^{2}-r}\right)
$$

where the summation is understood to be over all odd primes $r<q$ with the exception of $r=p$. It is a straightforward matter, by summing a 
finite number of terms and then using integrals to bound the remainder, to arrive at

$$
\sum_{\text {odd prime } r} \frac{1}{r^{2}-r}<0.2733 .
$$

In addition we may use a result from a paper of Rosser and Schoenfeld [16], (3.20), to see that

$$
\sum_{\text {odd prime } r}^{N} \frac{1}{r}<\log \log N
$$

These inequalities permit the following simplification:

$$
\begin{aligned}
& \sum_{\substack{\text { odd prime } \\
r<q}} P(p r \mid h) \\
& \quad<\frac{0.307}{p^{2}-p}-\frac{1.12}{p^{2}(p-1)^{2}}+\frac{164+597 \log \log q}{q^{0.125}(p-1)}-\frac{597}{q^{0.125}(p-1)^{2}} .
\end{aligned}
$$

Next we wish to determine an upper bound for $P\left(p^{2} \mid h\right)$. Since $p \nmid q-1$ it follows that $\sigma\left(p^{2}\right)=1$. Making use of this in (2.1) with $v=p^{2}$ and $w=1$ gives us

$$
\sum_{\substack{A \equiv 0\left(\bmod p^{2}\right) \\ A \text { odd }}} N_{q}(A, B)<\frac{0.74 q}{p^{2}-p}\left(1+\frac{264 p^{2}}{q^{0.125}}\right) .
$$

Since $p \nmid q-1$ all of the elliptic curves in the isomorphism classes above have cyclic $p$-Sylow groups and it follows that for each such curve fewer than $\# E\left(\mathbb{F}_{q}\right) / p^{2}$ of the points on the curve will be associated with an $M \in S_{q}^{\prime}$ with $h_{M} \equiv 0\left(\bmod p^{2}\right)$. We conclude that

$$
P\left(p^{2} \mid h\right)<\frac{1.12}{p^{4}-p^{3}}+\frac{294}{q^{0.125}\left(p^{2}-p\right)} .
$$

Combining our pieces shows that

$$
\begin{aligned}
P(h=p)> & \frac{0.867}{p^{2}-p}-\frac{234}{q^{0.125} p}-\frac{1.12}{p^{4}-p^{3}}-\frac{294}{q^{0.125}\left(p^{2}-p\right)}-\frac{0.307}{p^{2}-p} \\
& +\frac{1.12}{p^{2}(p-1)^{2}}-\frac{164+597 \log \log q}{q^{0.125}(p-1)}+\frac{597}{q^{0.125}(p-1)^{2}},
\end{aligned}
$$

which we simplify to get

$$
P(h=p)>\frac{1}{p^{2}-p}\left(0.56-\frac{p(597 \log \log q+398)}{q^{0.125}}+\frac{1.12}{p^{2}(p-1)}\right) .
$$

Since $(597 \log \log q+398) / q^{0.095}<0.236$ for $q>e^{100}$ we see that we may 
replace the above bound with

$$
\frac{1}{p^{2}-p}\left(0.56-\frac{0.236 p}{q^{0.03}}+\frac{1.12}{p^{2}(p-1)}\right)
$$

as was required.

We next treat the case $p \mid q-1$. Referring to Theorem 3.1 we see that

$$
P(h \equiv 0(\bmod p))>\frac{0.867(p+1)}{p^{3}}-\frac{234}{q^{0.125} p}-\frac{150}{q^{0.125} p^{1.75}} .
$$

All that remains is the determination of $P\left(p^{2} \mid h\right)$. It is in this calculation where the complication due to a $p$-rank of 2 rears its ugly head. As before, we subtract off an amount due to the case where the elliptic curve has a $p$-Sylow group that is cyclic and of order at least $p^{2}$. But we also need to discuss the situation where the $p$-Sylow group has a $p$-rank of 2 .

If $E\left(\mathbb{F}_{q}\right) \cong C_{p^{n}}$ with $n \geq 2$ or if $E\left(\mathbb{F}_{q}\right) \cong C_{p} \times C_{p}$ then fewer than $\# E / p^{2}$ of the points $\mathcal{P}$ on $E$ will be associated with an $M \in S$ such that $p^{2} \mid h_{M}$. If $E\left(\mathbb{F}_{q}\right) \cong C_{p^{m}} \times C_{p}$ with $m \geq 2$ then less than $\# E / p$ of the points $\mathcal{P}$ on $E$ will be associated with an $M \in S$ such that $p^{2} \mid h_{M}$. If $E\left(\mathbb{F}_{q}\right) \cong C_{p^{m}} \times C_{p^{n}}$ with $m \geq n \geq 2$ then all of the finite points $\mathcal{P}$ on $E$ will be associated with an $M \in S$ such that $p^{2} \mid h_{M}$. We shall use (2.1) three times, once each with $(v, w)=\left(p^{2}, 1\right),(p, p)$ and $\left(1, p^{2}\right)$. Both of the latter two cases are subsets of the preceding cases and we shall correct our results against overcounting. We obtain

$$
\begin{aligned}
P\left(p^{2} \mid h\right)< & \frac{1.12}{p^{4}-p^{3}}\left(1+\frac{264 p^{3}}{q^{0.125}}\right)+\frac{1.12}{p^{5}}\left(1+\frac{264 p^{1.25} p}{q^{0.125}}\right) \\
& +\frac{1.12(p-1)}{p^{7}}\left(1+\frac{132 p^{2.5}}{q^{0.125}}\right) \\
< & \frac{1}{p^{2}-p}\left(\frac{1.12(p+1)}{p^{3}}+\frac{296 p}{q^{0.125}}+0.0006\right),
\end{aligned}
$$

since

$$
\frac{296 p^{0.25}}{q^{0.125} p}+\frac{148 p^{0.5}}{q^{0.125} p^{2}}<0.0006
$$

Combining our many pieces for the $p \mid q-1$ case, noting that the bound for $P(p r \mid h)$ is the same as we used previously, shows that

$$
\begin{aligned}
P(h=p)> & \frac{1}{p^{2}-p}\left(\frac{0.867\left(p^{2}-1\right)}{p^{2}}-\frac{234(p-1)}{q^{0.125}}-\frac{150(p-1)}{q^{0.125} p^{0.75}}\right) \\
& -\frac{1}{p^{2}-p}\left(0.307-\frac{1.12}{p(p-1)}\right.
\end{aligned}
$$




$$
\begin{aligned}
& \left.+\frac{(164+597 \log \log q) p}{q^{0.125}}-\frac{597 p}{q^{0.125}(p-1)}\right) \\
& -\frac{1}{p^{2}-p}\left(\frac{1.12(p+1)}{p^{3}}+\frac{296 p}{q^{0.125}}+0.0006\right)
\end{aligned}
$$

which gives us

$$
P(h=p)>\frac{1}{p^{2}-p}\left(0.559-\frac{0.263 p}{q^{0.03}}-\frac{0.867}{p^{2}}+\frac{1.12}{p^{3}(p-1)}\right)
$$

as

$$
\frac{597 \log \log q+694+150 p^{-0.75}}{q^{0.095}}<0.263 .
$$

This concludes our proof.

Corollary 3.6. Let $q>e^{100}$ be prime. Let $S$ be the set of all monic quartics $M=T^{4}+a T^{2}+b T+c \in \mathbb{F}_{q}[T]$ (for some $a, b, c \in \mathbb{F}_{q}$ ) that are divisible by some irreducible cubic in $\mathbb{F}_{q}[T]$ and that have no cubic term. Let $h_{M}$ be the ideal class number of $\mathbb{F}_{q}(T, \sqrt{M(T)})$ and write $P(h=p)$ as shorthand for $\#\left\{M \in S_{q}: h_{M}=p\right\} / \# S_{q}$. Then

$$
\begin{array}{ll}
9.78 \%<P(h=3)<18.7 \% & \text { if } 3 \nmid q-1, \\
2.56 \%<P(h=5)<5.61 \% & \text { if } 5 \nmid q-1, \\
1.14 \%<P(h=7)<2.67 \% & \text { if } 7 \nmid q-1, \\
0.39 \%<P(h=11)<1.02 \% & \text { if } 11 \nmid q-1, \\
8.09 \%<P(h=3)<18.7 \% & \text { if } 3 \mid q-1, \\
2.48 \%<P(h=5)<5.61 \% & \text { if } 5 \mid q-1, \\
1.07 \%<P(h=7)<2.67 \% & \text { if } 7 \mid q-1, \\
0.37 \%<P(h=11)<1.02 \% & \text { if } 11 \mid q-1 .
\end{array}
$$

Proof. These statements follow immediately from (3.4) and (3.5).

In the number field situation one expects (see the heuristics of Cohen and Lenstra [4]) that the ideal class number of $\mathbb{Q}(\sqrt{p})$ is 1 for about $75.4 \%$ of all primes $p$. The function field case in which we find ourselves is somewhat different and it is worth mentioning that computational results [7] suggest very strongly that there is no limit for $P(h=1)$ as $q \rightarrow \infty$. Rather, what is observed is that the probability depends crucially on the primes dividing $q-1$. What we do expect, however, is that, no matter what the divisibility of $q-1$, if $q$ is sufficiently large $(q>10000$ should suffice) then $74.5 \%<P(h=1)<76.5 \%$. In the following theorem we prove bounds that are considerably looser.

Theorem 3.7. Let $q>e^{100}$ be prime. Define $S_{q}$ to be the set of all monic quartics $M \in \mathbb{F}_{q}[T]$ that are divisible by an irreducible cubic in $\mathbb{F}_{q}[T]$. Let 
$h_{M}$ be the ideal class number of $\mathbb{F}_{q}(T, \sqrt{M(T)})$ and write $P(h=1)$ as shorthand for $\#\left\{M \in S_{q}: h_{M}=1\right\} / \# S_{q}$. Then

$$
70 \%<P(h=1)<84 \% \text {. }
$$

Proof. From Theorem 3.1 the number of monic quartics $M \in S_{q}$ that have the ideal class number of $\mathbb{F}_{q}(T, \sqrt{M(T)})$ divisible by some $p$ is bounded from above by

$$
\sum_{p \text { odd prime }} q^{4}\left(\frac{0.371}{p(p-1)}+\frac{99}{q^{0.125} p}+\frac{78}{q^{0.125} p^{1.75}}\right) .
$$

We recall from the proof of Theorem 3.3 that

$$
\sum_{p \text { odd prime }} \frac{1}{p^{2}-p}<0.2733 \text { and } \sum_{p \text { odd prime }}^{N} \frac{1}{p}<\log \log N
$$

and easily obtain, as well,

$$
\sum_{p \text { odd prime }} \frac{1}{p^{1.75}}<0.31
$$

Combining these results, for $q>e^{100}$, gives the number of quartics $M \in S_{q}$ that have some prime dividing the ideal class number as being bounded from above by $0.1032 q^{4}$. But this quantity counts $M$ more than once whenever $h_{M}$ is divisible by more than one prime. We shall attempt a small correction of this by subtracting out the number of $M$ with $h_{M}$ divisible by $p r$. To do this we will require lower bounds for $P(p r \mid h)$ where $p$ and $r$ are distinct odd primes. From (2.2) we have, when $p r<q^{0.07}$,

$$
\sum_{\substack{A B \equiv 0(\bmod p r) \\ A B \text { odd }}} N_{q}(A, B)>\frac{0.58 q p r}{\left(p^{2}-1\right)\left(r^{2}-1\right)}\left(1-\frac{528 p r}{q^{0.125}}\right) .
$$

Since every elliptic group in the isomorphism classes above has at least $\# E\left(\mathbb{F}_{q}\right) /(p r)-1$ values of associated $M \in S_{q}^{\prime}$ with $h_{M} \equiv 0(\bmod p r)$ we can obtain the following lower bound:

$$
\frac{0.289 q^{3}}{\left(p^{2}-1\right)\left(r^{2}-1\right)}-\frac{154 p r q^{3}}{q^{0.125}\left(p^{2}-1\right)\left(r^{2}-1\right)}
$$

for the number of $M \in S_{q}^{\prime}$ with $h_{M} \equiv 0(\bmod p r)$. A short computation, summing over all products $p r<500$ of distinct odd primes $p$ and $r$, gives us a lower bound of $0.0037 q^{4}$ for the number of $M \in S_{q}$ whose class numbers are divisible by at least 2 distinct primes. Subtracting this from our earlier result shows that there are at most $0.0995 q^{4}$ values of $M \in S_{q}$ whose ideal class number is divisible by some prime. Since $\# S_{q}=\left(q^{4}-q^{2}\right) / 3$ it follows that at least $70 \%$ of the $M \in S_{q}$ must satisfy $h_{M}=1$. 
To obtain the upper bound we use Theorem 3.1 and Corollary 3.6 to see that

$$
\begin{gathered}
P(h=1)<1-P(h \equiv 0(\bmod 3))-P(h=5)-P(h=7), \\
P(h=1)<1-0.128-0.0248-0.0107<0.84,
\end{gathered}
$$

as required.

Previously mentioned conjectures [7] lead to the prediction that we should expect

$$
\lim _{\substack{q \rightarrow \infty \\ q \equiv 1(\bmod p)}} P(p-\text { rank }=2)=\frac{1}{p^{3}\left(p^{2}-1\right)} .
$$

Our final theorem proves bounds which create an interval approximately centered on the expected result.

TheOREM 3.8. Let $q>e^{100}$ be prime. Let $p<q^{0.03}$ be an odd prime. Define $S_{q}$ to be the set of all monic quartics $M \in \mathbb{F}_{q}[T]$ that are divisible by an irreducible cubic in $\mathbb{F}_{q}[T]$. Let $r_{p}(M)$ be the p-rank of the ideal class group of $\mathbb{F}_{q}(T, \sqrt{M(T)})$ and write $P\left(r_{p}=2\right)$ as shorthand for $\#\left\{M \in S_{q}\right.$ : $\left.r_{p}(M)=2\right\} / \# S_{q}$. Then $P\left(r_{p}=2\right)=0$ if $p \nmid q-1$ and if $p \mid q-1$ we have

$$
0.85 / p^{5}<P\left(r_{p}=2\right)<1.14 / p^{5} .
$$

Pr o o f. If $p \nmid q-1$ then we will refer to the remark following (2.2) together with Theorem 2.3 to conclude that a $p$-rank of 2 is impossible here. Substituting $w=p$ and $v=1$ into (2.1) and (2.2) gives us the following bounds:

$$
\sum_{\substack{B \equiv 0(\bmod p) \\ A B \equiv 0\left(\bmod p^{2}\right) \\ A B \text { odd }}} N_{q}(A, B)<\frac{0.74 q}{p^{3}}+\frac{98 p^{1.25}}{q^{0.125} p^{3}}<\frac{0.74 q}{p^{3}}+\frac{98}{q^{0.0875} p^{3}}<\frac{0.756 q}{p^{3}}
$$

and

$$
\sum_{\substack{B \equiv 0(\bmod p) \\ A B \equiv 0\left(\bmod p^{2}\right) \\ A B \operatorname{odd}}} N_{q}(A, B)>\frac{0.58 q}{p^{3}}-\frac{77 p^{1.25}}{q^{0.125} p^{3}}>\frac{0.567 q}{p^{3}} .
$$

To translate the above bounds into the quartic case we will use Theorem 2.3 and we must determine, for an arbitrary abelian group $G$ of $p$-rank 2, how many elements $g \in G$ are such that the $G /\langle g\rangle$ is non-cyclic. If we write $G=\left\{a^{i} b^{j}: i, j \in \mathbb{Z}\right\}$ where $a$ and $b$ are independent and generate $G$, then it is easy to see that $G /\langle g\rangle$ is non-cyclic precisely when $g=a^{p x} b^{p y}$ for some integers $x$ and $y$. It then follows immediately from Theorem 2.3 and previous 
comments that

$$
\begin{aligned}
& \#\left\{M \in S_{q}: r_{p}(M)=2\right\}<q \frac{0.756 q}{p^{3}} \cdot \frac{q-1}{2}\left(\frac{q+2 \sqrt{q}+1}{p^{2}}-1\right)<\frac{0.378 q^{4}}{p^{5}} \\
& \#\left\{M \in S_{q}: r_{p}(M)=2\right\}>q\left(\frac{0.557 q}{p^{3}}-3\right) \frac{q-1}{2}\left(\frac{q-2 \sqrt{q}+1}{p^{2}}-1\right) \\
&>\frac{0.2834 q^{4}}{p^{5}}
\end{aligned}
$$

from which we also obtain

$$
0.85 q / p^{5}<P\left(r_{p}=2\right)<1.14 q / p^{5} .
$$

It is worth noting that when $p$ is very small in comparison to $q$ the above results can be sharpened slightly. For example, when $p=3$ and $q>e^{100}$ with $3 \mid q-1$ then we can show that the probability that the 3 -rank is 2 (for the ideal class group of $\mathbb{F}_{q}(T, \sqrt{M(T)})$ with $\left.M \in S_{q}\right)$ is between $0.86 / 3^{5}$ and $1.12 / 3^{5}$.

\section{References}

[1] E. Artin, Quadratische Körper im Gebiet der höheren Kongruenzen I, II, Math. Z. 19 (1924), 153-246.

[2] D. A. Buell, Class groups of quadratic fields II, Math. Comp. 48 (1987), 85-93.

[3] - The expectation of success using a Monte Carlo factoring method-some statistics on quadratic class numbers, ibid. 43 (1984), 313-327.

[4] H. Cohen and H. W. Lenstra, Jr., Heuristics on class groups of number fields, in: Number Theory (Noordwijkerhout, 1983), H. Jager (ed.), Lecture Notes in Math. 1068, Springer, Berlin, 1984, 33-62.

[5] M. Eichler, Introduction to the Theory of Algebraic Numbers and Functions, Academic Press, New York, 1966.

[6] E. Friedman and L. C. Washington, On the distribution of divisor class groups of curves over a finite field, in: Théorie des nombres (Québec, PQ, 1987), de Gruyter, Berlin, 1989, 227-239.

[7] C. Friesen, Class group frequencies of real quadratic function fields: The degree 4 case, Math. Comp. 69 (2000), 1213-1228.

[8] -, Bounds for the number of certain elliptic curves over finite fields, preprint, 1999.

[9] -, A special case of Cohen-Lenstra heuristics in function fields, in: Fifth Conference of the Canadian Number Theory Association, K. S. Williams and R. Gupta (eds.), CRM Proc. Lecture Notes 19, Amer. Math. Soc., Providence, RI, 1999, 99-105.

[10] C. Friesen and P. van Wamelen, Class numbers of real quadratic function fields, Acta Arith. 81 (1997), 45-55.

[11] C. F. Gauss, Disquisitiones Arithmeticae, Yale University Press; A. A. Clarke, New Haven, Connecticut, 1966.

[12] D. R. Hayes, Real quadratic function fields, in: Canad. Math. Soc. Proc. 7, 1985, 203-236. 
[13] H. Ichimura, Class numbers of real quadratic function fields of genus one, Finite Fields Appl. 3 (1997), 181-185.

[14] S. Kuroda, Table of class number $h(p)>1$ for quadratic fields $Q(\sqrt{p}), p \leq 2776817$, Math. Comp. 29 (1975), 335-336.

[15] M. Madan, Note on a problem of S. Chowla, J. Number Theory 2 (1970), 279-281.

[16] J. B. Rosser and L. Schoenfeld, Approximate formulas for some functions of prime numbers, Illinois J. Math. 6 (1962), 64-94.

[17] M. Saito and H. Wada, Tables of ideal class groups of real quadratic fields, Proc. Japan Acad. Ser. A Math. Sci. 64 (1988), 347-349.

[18] R. Scheidler, A. Stein and H. C. Williams, Key-exchange in real quadratic congruence function fields, Des. Codes Cryptogr. 7 (1996), 153-174.

[19] T. A. Schmidt, Infinitely many real quadratic fields of class number one, J. Number Theory 54 (1995), 203-205.

[20] M. Tennenhouse and H. C. Williams, A note on class-number one in certain real quadratic and pure cubic fields, Math. Comp. 46 (1986), 333-336.

[21] J.-K. Yu, Toward a proof of the Cohen-Lenstra conjecture in the function field case, preprint, 1996.

Ohio State University at Marion

1465 Mt. Vernon Ave.

Marion, OH 43302, U.S.A.

E-mail: friesen.4@osu.edu

Received on 19.8.1999

and in revised form 4.5.2000 